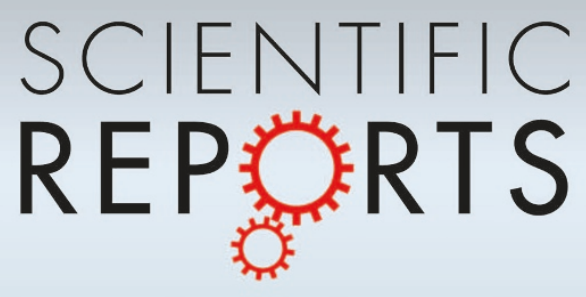

OPEN

SUBJECT AREAS:

$X$ - RAY

CRYSTALLOGRAPHY

SUPRAMOLECULAR ASSEMBLY

Received

22 August 2014

Accepted

29 October 2014

Published

14 November 2014

Correspondence and requests for materials should be addressed to

Y.S. (yuequan74@ yahoo.com; yshen@ nankai.edu.cn)

\section{Structural Insight into the Central Element Assembly of the Synaptonemal Complex}

\author{
Jing $\mathrm{Lu}^{1,2}$, Yanling $\mathrm{G} u^{1,2}$, Jianrong Feng ${ }^{1,2}$, Weihong Zhou' ${ }^{2}$, Xue Yang ${ }^{1} \&$ Yuequan Shen ${ }^{1,2,3}$
}

\begin{abstract}
${ }^{1}$ State Key Laboratory of Medicinal Chemical Biology, Nankai University, 94 Weijin Road, Tianjin 300071, China, ${ }^{2}$ College of Life Sciences, Nankai University, 94 Weijin Road, Tianjin 300071, China, ${ }^{3}$ Synergetic Innovation Center of Chemical Science and Engineering, 94 Weijin Road, Tianjin 300071, China.
\end{abstract}

\begin{abstract}
The key step in meiosis is synaptonemal complex formation, which mediates homologous chromosome alignment and synapsis. False pairing between homologous chromosomes produces infertility. Here, we present a crystal structure of the mouse meiosis-specific protein SYCE3, which is a component of the synaptonemal complex central element. Our studies show that functional SYCE3 most likely forms a dimer or higher order oligomer in cells. Furthermore, we demonstrate that the SYCE3 N-helix interacts with the SYCE1 C-helix, which is another central element component. Our results suggest that helical packing may mediate intra- or inter-association of each central element protein component, thereby playing an essential role in forming the synaptonemal complex central elements.
\end{abstract}

exual reproduction occurs in eukaryotes through meiosis, a process by which a diploid germ-line cell divides, resulting in the formation of haploid cells. This process involves one round of DNA replication and two rounds of cell division. Errors during meiosis can lead to disorders that result from an abnormal number of chromosomes ${ }^{1-3}$. Prior to the first division, the key step of meiosis is that two homologous chromosomes pair and that a synaptonemal complex (SC) develops between them. Once synapsis is complete, the homologous chromosomes undergo recombination ${ }^{4-7}$.

The synaptonemal complex is a ladder-like structure consisting of three components: lateral elements (LEs), central elements (CEs), and transverse filaments (TFs) ${ }^{7}$. These components are composed of meiosis-specific proteins. When homologous chromosomes begin synapsis, SYCP2 and SYCP3 form the axial elements (AEs) ${ }^{8}$, and when AEs are associated with a pair of sister chromatids, the mature AEs are called lateral elements ${ }^{7}$. In the case of SYCP2 lacking its coiled-coil domain, which is required for binding to SYCP3, male mice are sterile because of the failure of SC formation, whereas females are subfertile and have reduced litter size ${ }^{9}$. However, in mice lacking SYCP3, female mice exhibit aneuploidy because of defective meiotic chromosome segregation ${ }^{10}$. The TF is a physical linkage between the LE and the CE. SYCP1, a major component of TFs, is a long coiled-coil protein with $\mathrm{N}$ - and C-terminal globular domains ${ }^{11-13}$. Mutant SYCP1 with a modified length of the central alphahelical domain influences the SC width, and deletions of the globular heads result in the failure of SC assembly ${ }^{14}$.

In the last few years, four proteins of CEs have been identified: SYCE1, SYCE2, SYCE3, and TEX12. All of these proteins localize exclusively to the central element of the SC and contain predicated alpha-helical domains ${ }^{15-17}$. Both SYCE1 and SYCE2 directly interact with SYCP1 in vivo and in vitro. SYCP1 can play a role in recruiting SYCE1 and SYCE2 to the polycomplexes ${ }^{15}$, and in syce $1^{-1-}$ mutant mice, AEs are formed but homologous chromosomes fail to synapse ${ }^{18}$. In syce $2^{-/-}$mutant mice, the SC fails to form, except for small regions, suggesting that SYCE2 could be involved in SC extension ${ }^{19}$.

SYCE3, a newly identified component of the central element, is reported to co-localize with SYCE1 and SYCP1. SYCE3-knockout mice exhibit both male and female infertility. In syce $3^{-/-}$spermatocytes, SYCP1 displays a weak pattern at AEs, whereas SYCE1 and SYCE2 are completely absent, indicating that SYCE3 assembles to the CE prior to SYCE1 and SYCE2. SYCE3 is absent from the AEs in sycp $1^{-1-}$ mice. However, SYCE3 localizes to the chromosome axes in mice lacking SYCE1, SYCE2, or TEX12, suggesting that SYCE3 loading requires SYCP ${ }^{16}$.

The correct formation of the synaptonemal complex is crucial for homologous chromosome recombination and segregation. To further study the molecular basis of central element assembly, we determined the crystal structure of SYCE3 and revealed the extensive interactions involved in its dimer formation. Furthermore, we found that the interaction between SYCE3 and SYCE1 occurs through the N-terminal helix of SYCE3 and the Cterminal helix of SYCE1. Our findings provide evidence that the helical packing of SC proteins contributes to synaptonemal complex assembly. 


\section{Results}

Overall SYCE3 structure. The SYCE3 crystal structure was determined to a $1.9 \AA$ resolution using the single-wavelength anomalous dispersion method. As predicted, the SYCE3 structure consists of two helices: the N-helix (aa 1-48) and C-helix (aa 55-88). Except for the loop connecting the two helices, SYCE3 exhibits a clear electron density (Figure 1A). The protein crystals belong to the primitive rhombohedral space group $R 3$ with two independent molecules in each asymmetric unit that are related by 2 -fold noncrystallographic symmetry (Figure 1B). The SYCE3 primary sequence is highly conserved among mammals (Figure 1C), which indicates that both the three-dimensional structure and biological function are conserved.

Dimer packing in the SYCE3 structure. The crystal structure shows that two SYCE3 molecules interact closely (Figure 1B). The interaction between two SYCE3 molecules mainly involves an intensive network of hydrophobic contacts, hydrogen bonds, and salt bridges (Figure 2A). First, the two protomers primarily interact through a salt bridge. Glu74 from one protomer interacts with Lys34 from another protomer and forms a hydrogen bond with the Glu31 OE2 atom from another protomer (Figure 2A-4). Second, the Phe66 side chain from one protomer is buried by the hydrophobic cleft that consists of side chains from another protomer, which is composed of residues Leu21, Leu25, Leu28, and Leu80 (Figure 2A-5). Third, Trp77 is surrounded by a hydrophobic patch consisting of residues Ile35, Ala39, and Met42; Trp77 also forms a hydrogen bond with Asn38 from another protomer (Figure 2A-3). Fourth, Thr84 forms hydrophobic interactions with Leu62 and Ala65 (Figure 2A-1). Fifth, an Asn68 from one protomer forms two hydrogen bonds with another protomer through the side chain Glu79 OE2 atom and Glu83 OE2 atom. A hydrogen bond also forms between the Glu72 OE2 atom and Glu79 OE1 atom from each protomer (Figure 2A-2). These extensive interactions indicate highly stable dimer formation.

To examine the dimer status of SYCE3 in solution, we performed analytical ultracentrifugation. The results confirm that SYCE3 forms a homogenous dimer in solution with a molecular mass of approximately $20.5 \mathrm{kDa}$ (Figure 2B). Furthermore, to validate SYCE3 homo-

A
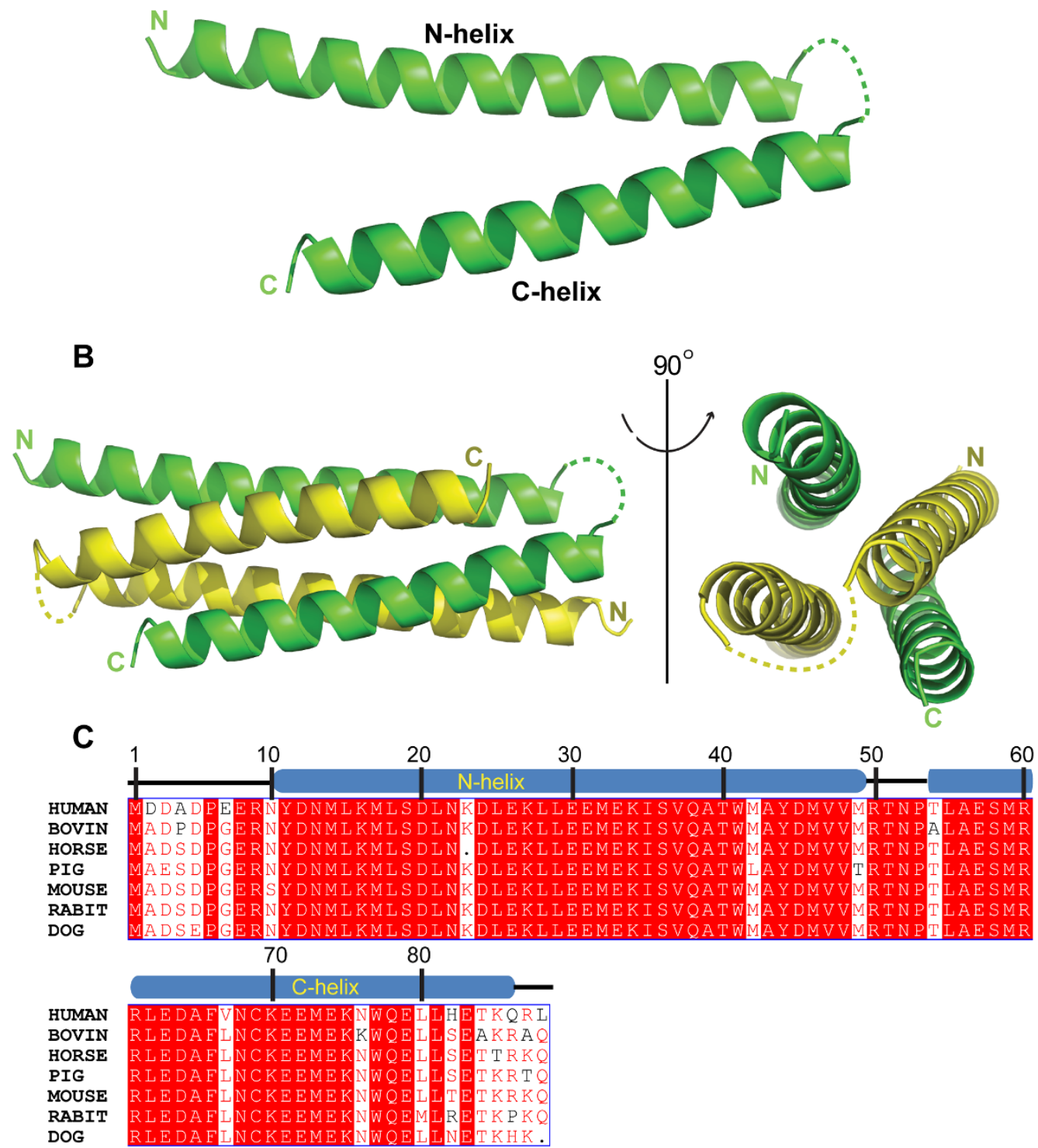

Figure 1 SYCE3 crystal structure. (A) A cartoon representation of the SYCE3 molecular structure. (B) A cartoon representation of the SYCE3 dimer structure. The two SYCE3 molecules are shown in yellow and green. The dotted lines represent the region that was not resolved in this structure. (C) A multiple sequence alignment for full-length SYCE3 among various mammals. The SYCE3 primary sequence is highly conserved among mammals. 
A
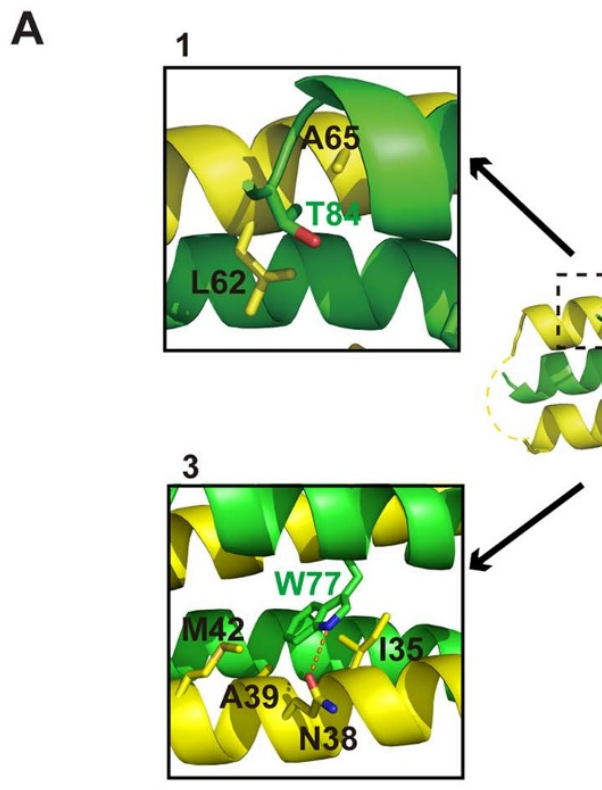

2
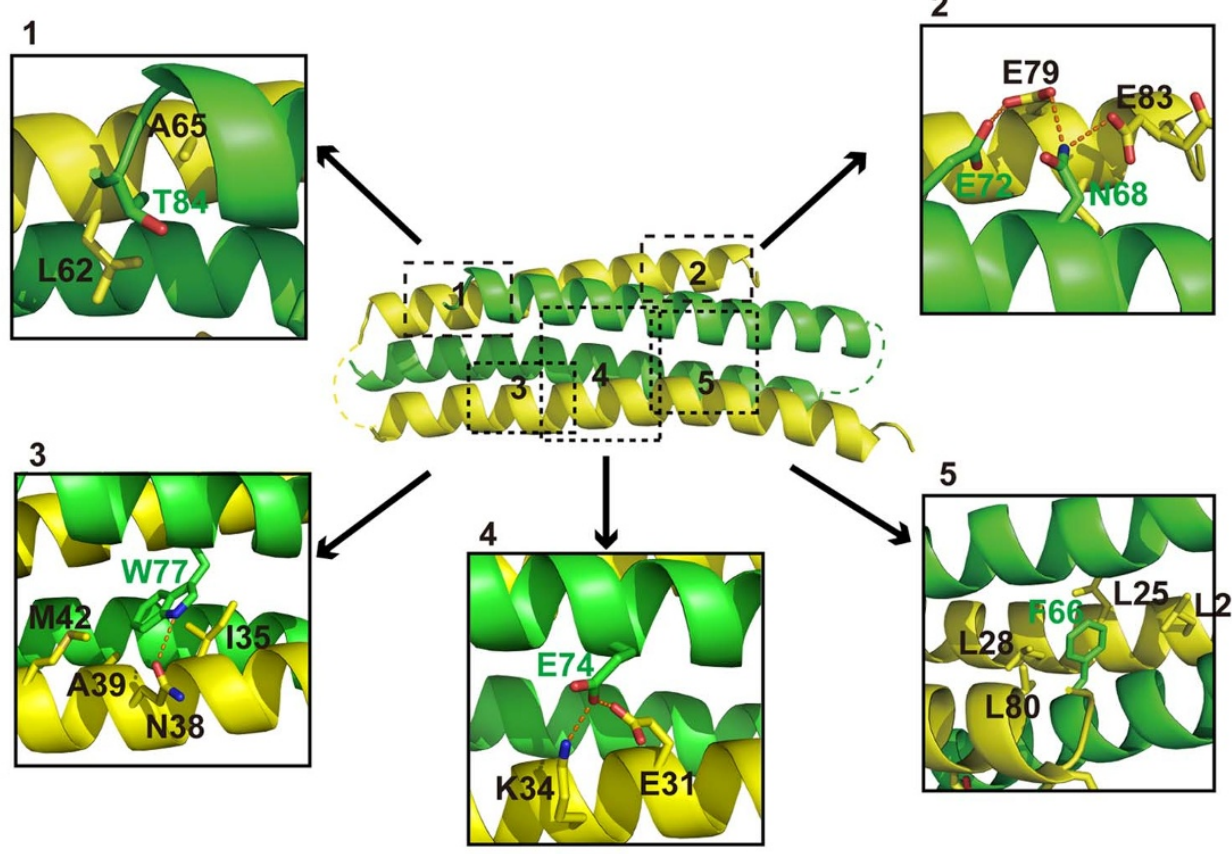

5

\section{B}

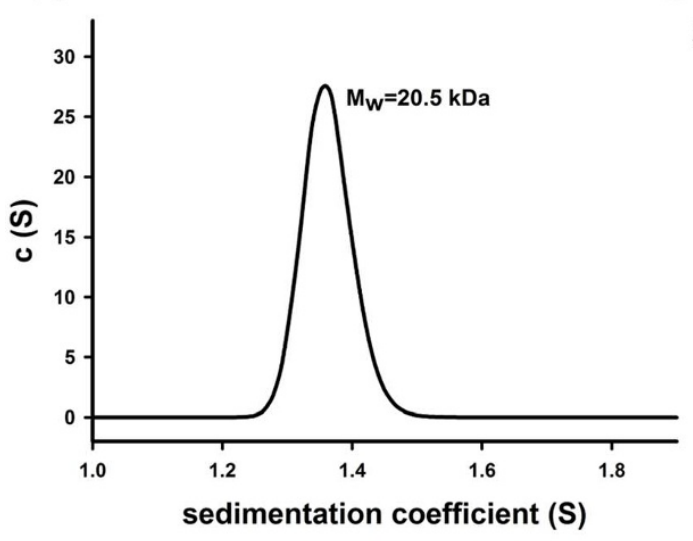

C

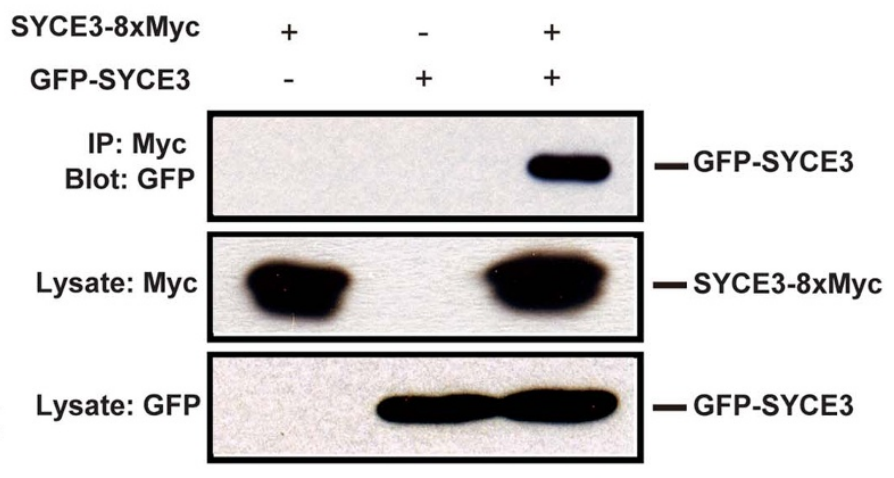

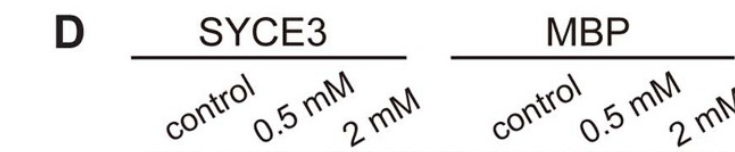

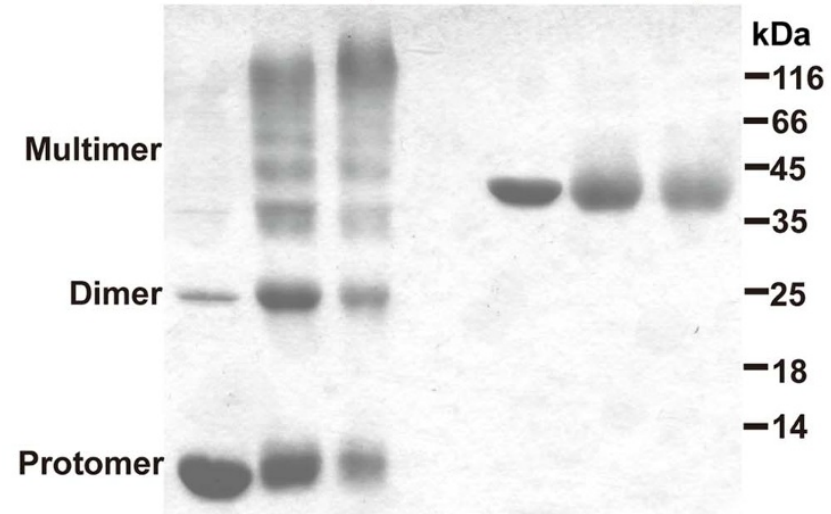

Figure $2 \mid$ SYCE3 dimeric packing. (A) Detailed interactions between two SYCE3 molecules in the SYCE3 dimer. The residues involved in dimer packing are boxed. The relevant amino acid residues are numbered, and the hydrogen bonds are shown as red dashed lines. (B) An analytical ultracentrifugation sedimentation velocity analysis of the purified SYCE3 protein. The calculated molecular weight is $20.5 \mathrm{kDa}$. (C) Co-immunoprecipitation assays for SYCE3 homodimerization. COS-7 cells were transfected with EGFP-SYCE3 and SYCE3-8 $\times$ Myc. The resulting complexes were analyzed using SDS-PAGE and Western blotting. (D) A cross-linking experiment for the purified SYCE3 protein. The final concentration ( $0.5 \mathrm{mM}$ and $2 \mathrm{mM})$ of DSG in the reaction mixture is indicated. Full-length blots/gels are presented in Supplementary Figure 1. 
A

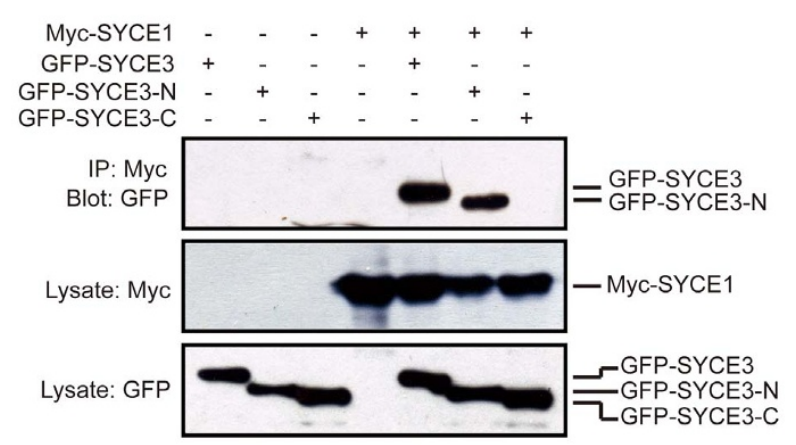

B

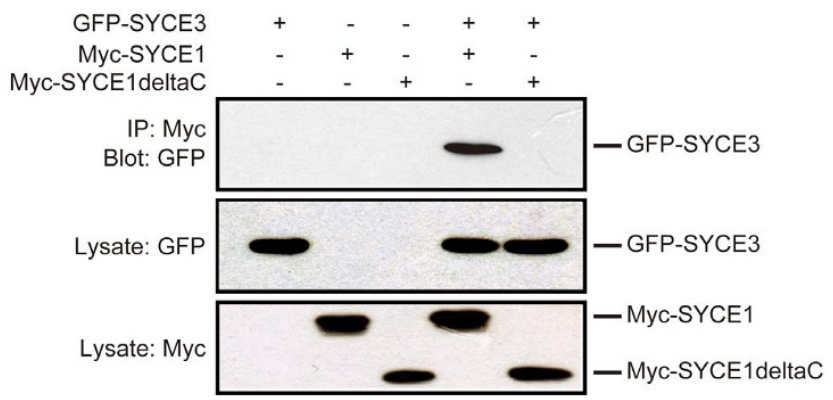

Figure $3 \mid$ Interactions between SYCE3 and SYCE1. (A) COS-7 cells were transfected with Myc-SYCE1 and the respective full-length SYCE3, SYCE3 N-helix, and SYCE3 C-helix EGFP fusion constructs. Myc-SYCE1 can bind the full-length SYCE3 and SYCE3 N-helix. The SYCE3 C-helix does not bind SYCE1. (B) COS-7 cells were transfected with EGFP-SYCE3 and the respective Myc fusion constructs composed of full-length SYCE1 and SYCE1 without C-helix. The results show that SYCE1 without the C-helix does not bind SYCE3. Full-length blots/gels are presented in Supplementary Figure 1.

dimerization or homo-oligomerization in cells, we performed cotransfection and immunoprecipitation experiments in somatic cells, which do not express meiosis-specific proteins ${ }^{20}$. Because the MycSYCE3 fusion protein did not bind the anti-Myc coupled protein A/ $\mathrm{G}$ beads, we modified pcDNA4/myc-His A by inserting $7 \times$ Myc tags between the SYCE3 gene and original Myc tag. The SYCE3-8 $\times$ Myc and EGFP-SYCE3 fusion constructs were then co-transfected into COS-7 cells. The results show that SYCE3-8 $\times$ Myc can successfully pull down GFP-SYCE3 (Figure 2C), which indicates that SYCE3 can form a dimer in cells. To verify whether SYCE3 can form higher order oligomers, we performed a cross-linking experiment using purified SYCE3. The results show that SYCE3 can form a dimer or multimer after treatment with disuccinimidyl glutarate (DSG) (Figure 2D), which suggests that SYCE3 might form higher order oligomers in cells.

The interaction between SYCE3 and SYCE1. A previous report indicated that SYCE3 co-localizes with SYCE1 and aids in recruiting SYCE1 to SYCP1 ${ }^{16}$; thus, we performed co-IP experiments to further study the interaction between SYCE3 and SYCE1. To examine which SYCE3 helix interacts with SYCE1, we co-transfected constructs encoding EGFP-tagged fusion proteins composed of the full-length SYCE3, SYCE3 N-helix (aa1-52), or SYCE3 C-helix (aa 53-88) and a construct encoding Myc-tagged SYCE1 into COS-7 cells. Our data show that both the full-length SYCE3 and SYCE3 N-helix interacts with SYCE1; however, we did not detect an interaction between the SYCE3 C-helix and SYCE1 (Figure 3A).
We then attempted to map the portion of SYCE1 involved in binding SYCE3. We co-transfected Myc-tagged fusion constructs composed of full-length SYCE1 or SYCE1deltaC (a mutant of fulllength SYCE1 with the C-terminal aa 220-329 deleted) and EGFPtagged fusion constructs composed of full-length SYCE3 into COS-7 cells. We found that deleting the C-terminal portion of SYCE1 significantly weakened the association between SYCE1 and SYCE3 (Figure 3B). Taken together, our results suggest that the association between SYCE3 and SYCE1 is mainly involved in interactions between the SYCE3 N-terminal helix and SYCE1 C-terminal helix.

\section{Discussion}

The key step in meiosis is correct synaptonemal complex assembly. Several SC proteins have been identified in recent years; SYCE1, SYCE2, SYCE3, and TEX12 are SC central element proteins. Extensive studies have demonstrated that the CE proteins are crucial for SC assembly ${ }^{15-17}$. Our results, for the first time, provide structural information on the $\mathrm{CE}$ proteins and important insights into the central elements' assembly mechanism.

Secondary structure predictions for SYCE1, SYCE2, SYCE3, and TEX12 showed that they form helix-like structural features. In our SYCE3 structure, the extensive interactions between helices of two SYCE3 molecules indicate highly stable dimer formation (Figure 2A). This structure is consistent with our co-immunoprecipitation assay results from transfected COS-7 cells, which show that SYCE3 may function as a dimer or higher-order oligomer in cells during SC assembly. Previous reports indicate that SYCE3 and SYCE1 co-localize along synapsed chromosomes in zygotene and pachytene spermatocytes ${ }^{16}$. Our co-IP assay results demonstrate that SYCE3 can bind SYCE1. SYCE2 and TEX12 share similar localization patterns with SYCE1 and SYCE3 in the $\mathrm{CE}^{17}$, and previous reports indicate that SYCE2 co-localizes and co-precipitates with TEX12 during meiotic cell division ${ }^{17}$. Owen et al proposed an SYCE2-TEX12 equimolar hetero-octamer model ${ }^{21}$; in this model, SYCE2 molecules form a tetramer mediated by the central $\alpha$-helices, and TEX12 molecules form a dimer mediated by the central $\alpha$-helices. Further, the SYCE2 tetramer binds two TEX12 dimers through helical packing interactions between SYCE2 and TEX12, which forms a stable higher-order structure.

Based on our study, the SYCE3 N-helix participates in SYCE1 binding; however, the SYCE3 C-helix and SYCE1 do not interact (Figure 3A). We also mapped the SYCE1 C-terminal helix, which is crucial for binding between SYCE3 and SYCE1 (Figure 3B). Based on our structural and biochemical studies, we propose that the SYCE3 dimer binds two or more SYCE1 moieties via interactions between the SYCE3 N-helix and SYCE1 C-terminal helix. In the recently determined SYCP3 structure, four SYCP3 helical chains form a tetramer composed of helix bundles and coiled-coil motifs. The extensive network of hydrophobic contacts and salt bridges in the two helical bundles contributes to the high stability of SYCP3 packing $^{22}$. These findings support the notion that the helical bundle is important for synaptonemal complex formation. Overall, the helical features of each CE protein and the inter-helix interaction-mediated association between different CE proteins indicate that helical packing mainly drives central element assembly.

Previous studies show that SYCE1 stabilizes the SYCP1 N-terminal interactions in the $\mathrm{CE}$, which was demonstrated through co-IP assays and immunofluorescence analyses ${ }^{15}$. SYCE1 also colocalizes and co-precipitates with SYCE $3^{16}$. Thus, it is reasonable to assume that SYCE1 functions as a bridge between SYCP1 and SYCE3. However, according to Schramm's research, in Syce $3^{-/-}$mice, SYCP1 localizes in a weak pattern at AEs, with both SYCE1 and SYCE2 being absent from the axes ${ }^{16}$. SYCE3 is likely required for initial CE assembly. However, interactions between the SYCP1 Nterminus and SYCE3 remain vague, and co-IP assays do not show that SYCE3 can bind the SYCP1 N-terminal region ${ }^{16}$. It is possible 
that additional undiscovered CE proteins may connect SYCP1 and SYCE3.

The CE proteins not only play a structural role in the SC but can also be regulated by other proteins. PLK1, a Polo-like kinase, is required for SYCP1, TEX12, and SYCE1 phosphorylation to promote SC disassembly during the G2/MI transition ${ }^{23}$. Studies have also predicted phosphorylation sites for SYCE3 that could be regulated by kinase activity ${ }^{16}$. Further studies will elucidate the structural and regulatory roles of CE proteins in SC assembly.

\section{Methods}

Protein expression and purification. Gene fragments encoding SYCE3 were PCR amplified from Mus musculus cDNAs and then cloned into the $\mathrm{pET}-\mathrm{M}$ vector, a derivative of pET32a (Novagen). The correctness of the constructs was confirmed by DNA sequencing. Escherichia coli BL21 CondonPlus cells harboring the expression plasmid for the fusion protein were grown at $37^{\circ} \mathrm{C}$ to an $\mathrm{OD}_{600}>0.6$ and then induced with $0.2 \mathrm{mM}$ isopropyl- $\beta$-D-thiogalactoside (IPTG) at $20^{\circ} \mathrm{C}$ for $16-18 \mathrm{~h}$. The cells were resuspended in $\mathrm{T}_{20} \mathrm{~N}_{300} \mathrm{I}_{20}$ buffer $(20 \mathrm{mM}$ Tris- $\mathrm{HCl}, \mathrm{pH} 8.0,300 \mathrm{mM}$ $\mathrm{NaCl}$, and $20 \mathrm{mM}$ imidazole) and then lysed by sonication. After centrifugation at $20,000 \times g$ for $40 \mathrm{~min}$, the supernatant was loaded directly onto a Ni-NTA agarose column (Qiagen) equilibrated with $\mathrm{T}_{20} \mathrm{~N}_{300} \mathrm{I}_{20}$ buffer. After washing the Ni-NTA column with 5 column volumes of equilibrating buffer, the $\mathrm{His}_{6}$-tagged protein was eluted with $\mathrm{T}_{20} \mathrm{~N}_{300} \mathrm{I}_{300}$ buffer. Subsequently, the protein elute was subjected to anionexchange chromatography (Hitrap Q FF; GE Healthcare) using $\mathrm{T}_{20} \mathrm{~N}_{60} \mathrm{E}_{1} \mathrm{D}_{1}$ buffer (20 mM Tris- $\mathrm{HCl}, \mathrm{pH} 8.0,60 \mathrm{mM} \mathrm{NaCl}, 1 \mathrm{mM} \mathrm{EDTA}, 1 \mathrm{mM} \mathrm{DTT}$ ) from $6 \%$ to $50 \%$ $\mathrm{NaCl}$. The final target protein was loaded onto a HighLoad 26/600 Superdex 200 sizeexclusion column (GE Healthcare) and eluted with $\mathrm{T}_{20} \mathrm{~N}_{200} \mathrm{D}_{1}$ buffer (20 mM Tris$\mathrm{HCl}, \mathrm{pH}$ 8.0, $200 \mathrm{mM} \mathrm{NaCl}, 1 \mathrm{mM}$ DTT). The protein peak was identified by SDS-PAGE, harvested, and concentrated using a Centricon device (Millipore).

Crystallization and data collection. Crystals of wild-type and Se-Met-substituted SYCE3 were grown using sitting-drop vapor diffusion. SYCE3 was crystallized by combining $1 \mu \mathrm{L}$ of protein solution $(8.8 \mathrm{mg} / \mathrm{mL}$ in $20 \mathrm{mM}$ Tris- $\mathrm{HCl}, \mathrm{pH} 8.0$,

$200 \mathrm{mM} \mathrm{NaCl}, 1 \mathrm{mM}$ DTT) with an equal volume of well solution containing $0.1 \mathrm{M}$ citric acid, pH 3.5, 7\% 2-propanol, and 1\% PEG 20,000. The crystals were grown for approximately one week at $4{ }^{\circ} \mathrm{C}$ and frozen in cryoprotectant consisting of the well solution supplemented with $25 \%$ glycerol. Se-Met-substituted SYCE3 was crystallized by the combination of $1 \mu \mathrm{L}$ of protein solution $(3.7 \mathrm{mg} / \mathrm{mL}$ in $20 \mathrm{mM}$ Tris- $\mathrm{HCl}, \mathrm{pH}$ $8.0,200 \mathrm{mM} \mathrm{NaCl}, 1 \mathrm{mM}$ DTT) with an equal volume of well buffer containing $0.1 \mathrm{M}$ citric acid, $\mathrm{pH} 3.5,2 \%$ 2-propanol, and 5\% PEG 20,000. The crystals were grown for approximately one week at $4{ }^{\circ} \mathrm{C}$ and frozen in cryoprotectant consisting of the well solution supplemented with $25 \%$ glycerol.

The data for wild-type were collected at the beamline BL17U1 of the Shanghai Synchrotron Radiation Facility (SSRF), and single-wavelength anomalous data were collected for the Se-Met-substituted crystals at the elemental Se peak wavelength at the beamline BL-17A of the Photon Factory (Tsukuba, Japan) and then processed using the HKL2000 software ${ }^{24}$.

Structure determination and refinement. The SYCE3 crystal structure was determined by SAD methodology. The program HKL2MAP ${ }^{25}$ was used to search for Se sites, and the initial phases were then calculated using PHENIX software ${ }^{26}$. The model was built manually using the program $\mathrm{COOT}^{27}$. After the initial main-chain model was built, the wild-type data were applied to carry out an iterative refinement to assign all side chains using the program COOT until the free $\mathrm{R}$ factor was converged. The final structure had an $\boldsymbol{R}_{\text {crystal }}$ value of $21.47 \%$ and an $\boldsymbol{R}_{\text {free }}$ value of $23.45 \%$, with good geometry. The detailed data collection and refinement statistics are summarized in Table 1.

Analytical ultracentrifugation. Sedimentation velocity measurements were obtained using a Beckman/Coulter XL-I analytical ultracentrifuge with double-sector and sapphire windows. The SV experiments were conducted at $42,000 \mathrm{rpm}$ and $4{ }^{\circ} \mathrm{C}$ using interference light detection and double-sector centerpieces loaded with $1 \mathrm{mg} /$ $\mathrm{mL}$ protein. The sample was prepared in a buffer containing $20 \mathrm{mM}$ Tris- $\mathrm{HCl}, \mathrm{pH}$ 8.0 , and $200 \mathrm{mM} \mathrm{NaCl}$. The data were analyzed using the SEDFIT program ${ }^{28}$.

Cell culture and transfection. COS-7 (green monkey kidney) cells were cultured in Dulbecco's modified Eagle's medium (DMEM) supplemented with $10 \% \mathrm{v} / \mathrm{v}$ fetal bovine serum (FBS). The cells were maintained in a $95 \%$ air and $5 \% \mathrm{CO}_{2}$ environment at $37^{\circ} \mathrm{C}$.

Gene fragments corresponding to full-length SYCE3, SYCE3 N-helix (aa 1-52), and SYCE3 C-helix (aa 53-88) were cloned into the pEGFP-C1 vector (Clontech). The SYCE3 gene was also cloned into the pcDNA4/myc-His A vector (Invitrogen), and 7 Myc tags were inserted after the SYCE3 gene. This modified plasmid was named pcDNA4/8myc-His A. Gene fragments corresponding to full-length SYCE1 and SYCE1deltaC (aa 1-219) were cloned into the pCMV-Myc vector (Clontech).

Co-immunoprecipitation and Western blot analysis. Transfected COS-7 cells were collected in PBS buffer $\left(136 \mathrm{mM} \mathrm{NaCl}, 2.7 \mathrm{mM} \mathrm{KCl}, 10 \mathrm{mM} \mathrm{Na}_{2} \mathrm{HPO}_{4}, 1.5 \mathrm{mM}\right.$ $\mathrm{KH}_{2} \mathrm{PO}_{4}, \mathrm{pH}$ 7.4). Cells harvested from a $10-\mathrm{cm}$ culture dish were lysed in $500 \mu \mathrm{L}$ of

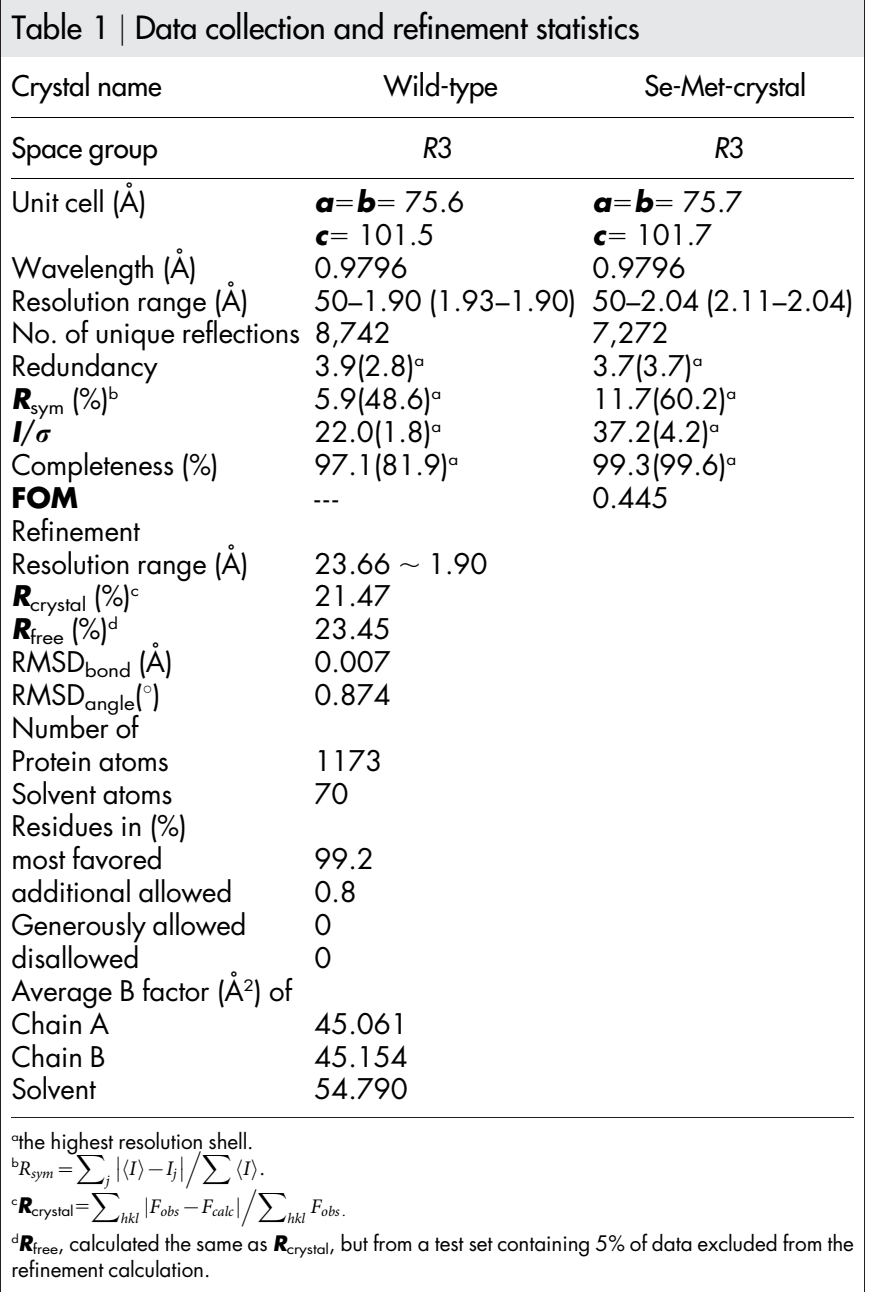

RIPA buffer (PBS containing 1.0\% Triton X-100, $0.5 \%$ sodium deoxycholate, $0.1 \%$ SDS, $1 \mathrm{mM} \beta$-glycerolphosphate, $1 \mathrm{mM} \mathrm{Na} \mathrm{VO}_{4}, 1 \mathrm{mM}$ EDTA, $\left.1 \mathrm{mM} \mathrm{EGTA}\right)$ containing Roche protease inhibitor cocktail. After incubation on ice for $1 \mathrm{~h}$, the lysate was centrifuged at $13,000 \times \mathrm{g}$ for $30 \mathrm{~min}$. A total of $40 \mu \mathrm{L}$ of a 1:1 slurry of protein A/G agarose (Pierce) was incubated with $2 \mu \mathrm{g}$ of anti-Myc antibody for $1 \mathrm{~h}$ and then washed twice with cold RIPA buffer. The cell extract supernatant was added to the antibody-coupled protein $\mathrm{A} / \mathrm{G}$ beads and was incubated for $3 \mathrm{~h}$ at $4{ }^{\circ} \mathrm{C}$. The beads were washed three times for $10 \mathrm{~min}$ with cold RIPA buffer, followed by elution with $40 \mu \mathrm{L}$ SDS-loading buffer. Subsequently, $5 \mu \mathrm{L}$ of the elute was loaded onto a $15 \%$ SDS-PAGE gel and transferred onto a PVDF membrane for Western blot analysis. The immunoblot analysis was conducted using a primary antibody against GFP. The protein-antibody complexes were detected using enhanced chemiluminescence.

Protein cross-linking. The purified proteins SYCE3 and maltose binding protein (MBP) were prepared in reaction buffer (20 mM HEPES, pH7.5, and $200 \mathrm{mM} \mathrm{NaCl})$, respectively. Disuccinimidyl glutarate was added to the protein sample at the final concentrations $0.5 \mathrm{mM}$ and $2 \mathrm{mM}$. Next, the reaction mixture was incubated at room temperature for 30 minutes. The samples were loaded onto a 15\% SDS-PAGE gel and stained with Coomassie brilliant blue.

1. Garcia-Cruz, R., Roig, I. \& Caldes, M. G. Maternal origin of the human aneuploidies. Are homolog synapsis and recombination to blame? Notes (learned) from the underbelly. Genome Dyn. 5, 128-136 (2009).

2. Handel, M. A. \& Schimenti, J. C. Genetics of mammalian meiosis: regulation, dynamics and impact on fertility. Nat. Rev. Genet. 11, 124-136 (2010).

3. Hassold, T. \& Hunt, P. To err (meiotically) is human: the genesis of human aneuploidy. Nat. Rev. Genet. 2, 280-291 (2001).

4. Fawcett, D. W. The fine structure of chromosomes in the meiotic prophase of vertebrate spermatocytes. J. Biophys. Biochem. Cytol. 2, 403-406 (1956).

5. Moses, M. J. Chromosomal structures in crayfish spermatocytes. J. Biophys. Biochem. Cytol. 2, 215-218 (1956).

6. Moses, M. J. Structure and function of the synaptonemal complex. Genetics. 61 , Suppl, 41-51 (1969). 
7. Page, S. L. \& Hawley, R. S. The genetics and molecular biology of the synaptonemal complex. Annu. Rev. Cell Dev. Biol. 20, 525-558 (2004).

8. Eijpe, M., Offenberg, H., Jessberger, R., Revenkova, E. \& Heyting, C. Meiotic cohesin REC8 marks the axial elements of rat synaptonemal complexes before cohesins SMC1beta and SMC3. J. Cell Biol. 160, 657-670 (2003).

9. Yang, F. et al. Mouse SYCP2 is required for synaptonemal complex assembly and chromosomal synapsis during male meiosis. J. Cell Biol. 173, 497-507 (2006).

10. Yuan, L. et al. Female germ cell aneuploidy and embryo death in mice lacking the meiosis-specific protein SCP3. Science 296, 1115-1118 (2002).

11. Meuwissen, R. L. et al. A coiled-coil related protein specific for synapsed regions of meiotic prophase chromosomes. EMBO J. 11, 5091-5100 (1992).

12. Liu, J. G. et al. Localization of the N-terminus of SCP1 to the central element of the synaptonemal complex and evidence for direct interactions between the Ntermini of SCP1 molecules organized head-to-head. Exp. Cell Res. 226, 11-19 (1996).

13. Schmekel, K. et al. Organization of SCP1 protein molecules within synaptonemal complexes of the rat. Exp. Cell Res. 226, 20-30 (1996).

14. Ollinger, R., Alsheimer, M. \& Benavente, R. Mammalian protein SCP1 forms synaptonemal complex-like structures in the absence of meiotic chromosomes. Mol. Biol. Cell 16, 212-217 (2005).

15. Costa, Y. et al. Two novel proteins recruited by synaptonemal complex protein 1 (SYCP1) are at the centre of meiosis. J. Cell Sci. 118, 2755-2762 (2005).

16. Schramm, S. et al. A novel mouse synaptonemal complex protein is essential for loading of central element proteins, recombination, and fertility. PLoS Genet. 7, e1002088 (2011)

17. Hamer, G. et al. Characterization of a novel meiosis-specific protein within the central element of the synaptonemal complex. J. Cell Sci. 119, 4025-4032 (2006).

18. Bolcun-Filas, E. et al. Mutation of the mouse Sycel gene disrupts synapsis and suggests a link between synaptonemal complex structural components and DNA repair. PLoS Genet. 5, e1000393 (2009).

19. Bolcun-Filas, E. et al. SYCE2 is required for synaptonemal complex assembly, double strand break repair, and homologous recombination. J. Cell Biol. 176, 741-747 (2007).

20. Stewart-Hutchinson, P. J., Hale, C. M., Wirtz, D. \& Hodzic, D. Structural requirements for the assembly of LINC complexes and their function in cellular mechanical stiffness. Exp. Cell Res. 314, 1892-1905 (2008).

21. Davies, O. R., Maman, J. D. \& Pellegrini, L. Structural analysis of the human SYCE2-TEX12 complex provides molecular insights into synaptonemal complex assembly. Open Biol. 2, 120099 (2012).

22. Syrjanen, J. L., Pellegrini, L. \& Davies, O. R. A molecular model for the role of SYCP3 in meiotic chromosome organisation. Elife. 3, e02963 (2014)

23. Jordan, P. W., Karppinen, J. \& Handel, M. A. Polo-like kinase is required for synaptonemal complex disassembly and phosphorylation in mouse spermatocytes. J. Cell Sci. 125, 5061-5072 (2012).

24. Otwinowski Z, M. W. Processing of X-ray diffraction data collected in oscillation mode. Method Enzymol. 276, 307-326 (1997).
25. Pape, T. \& Schneider, T. R. HKL2MAP: a graphical user interface for phasing with SHELX programs. J. Appl. Cryst. 37, 843-844 (2004).

26. Zwart, P. H. et al. Automated structure solution with the PHENIX suite. Methods Mol. Biol. 426, 419-435 (2008).

27. Emsley, P. \& Cowtan, K. Coot: model-building tools for molecular graphics. Acta Crystallogr. D Biol. Crystallogr. 60, 2126-2132 (2004).

28. Brown, P. H. \& Schuck, P. Macromolecular size-and-shape distributions by sedimentation velocity analytical ultracentrifugation. Biophys. J. 90, 4651-4661 (2006).

\section{Acknowledgments}

We are grateful to Dr. Zheng Wang for help in analytical ultracentrifugation experiment, to Dr. Zhijie Lin for help in protein expression and purification, to the staff at the beamline BL-17A at Photon Factory (Tsukuba, Japan) and at the beamline BL17U1 of the Shanghai Synchrotron Radiation Facility for excellent technical assistance during data collection This work was supported by the 973 Program (grants 2012CB917200 and 2013 CB910400 to YS; grant 2014CB910201 to XY), the Natural Science Foundation of China (grants 31370826 to YS, 31300628 to XY and 31170684 to WZ), Tianjin Basic Research Program (grant 14JCQNJ09300 to XY) and the Fundamental Research Funds for the Central Universities (grants 65142007 to YS; 65121016 to XY).

\section{Author contributions}

J.L., Y.G. and J.F. did protein purification; J.L. did crystallization, structure refinement co-IP and biochemical experiments; X.Y. did data collection and structure determination; W.Z. analyzed the data. J.L. and Y.S. designed the study and wrote the paper. All authors discussed the results and commented on the manuscript.

\section{Additional information}

Supplementary information accompanies this paper at http://www.nature.com/ scientificreports

Competing financial interests: The authors declare no competing financial interests.

How to cite this article: Lu, J. et al. Structural Insight into the Central Element Assembly of the Synaptonemal Complex. Sci. Rep. 4, 7059; DOI:10.1038/srep07059 (2014).

This work is licensed under a Creative Commons Attribution-NonCommercialShareAlike 4.0 International License. The images or other third party material in this article are included in the article's Creative Commons license, unless indicated otherwise in the credit line; if the material is not included under the Creative Commons license, users will need to obtain permission from the license holder in order to reproduce the material. To view a copy of this license, visit http:// creativecommons.org/licenses/by-nc-sa/4.0/ 